\title{
Is There an Association between Bacterial Vaginosis Infection and HIV-1 Infection Acquisition among Women Aged 18 - 35 Years in Soweto?
}

\author{
Nathan B. W. Chimbatata ${ }^{1,2}$ \\ ${ }^{1}$ School of Public Health, Witwatersrand University, Johannesburg, South Africa \\ ${ }^{2}$ Mzuzu University, Mzuzu, Malawi \\ Email: natchims@yahoo.com
}

How to cite this paper: Chimbatata, N.B.W. (2016) Is There an Association between Bacterial Vaginosis Infection and HIV-1 Infection Acquisition among Women Aged 18 - 35 Years in Soweto? Open Journal of Preventive Medicine, 6, 197-213.

http://dx.doi.org/10.4236/ojpm.2016.69019

Received: August 1, 2016

Accepted: September 19, 2016

Published: September 22, 2016

Copyright (๑) 2016 by authors and Scientific Research Publishing Inc. This work is licensed under the Creative Commons Attribution International License (CC BY 4.0).

http://creativecommons.org/licenses/by/4.0/

(c) (i) Open Access

\section{Abstract}

Background: Studies suggest that there is association between Bacterial Vaginosis and Human Immunodeficiency Virus infection but its temporal effect has not been sufficiently investigated. Methods: It is a secondary data analysis following a cohort study. It was carried out to describe Bacterial Vaginosis infection association to $\mathrm{Hu}$ man Immunodeficiency Virus acquisition. The primary cohort study registered 750 study participants. A gram stain slide prepared from a vaginal swab was used to measure Bacterial Vaginosis as the primary exposure. A score of 7 or above was considered positive for Bacterial Vaginosis. The determination of the dependent variable Human Immunodeficiency Virus infection was achieved through dual rapid tests which were confirmed by using a third generation ELISA. Incident Human Immunodeficiency Virus infection rate was calculated. To test significance, Kaplan Meier survival time analysis and log rank test were carried out. The association of Bacterial Vaginosis with Human Immunodeficiency Virus infection was investigated using Cox regression. Results: The baseline prevalence of Bacterial Vaginosis was 52\%, 95\% CI; 45 - 59. There were 21 Human Immunodeficiency Virus seroconversions in total of which 7 had no Bacterial Vaginosis results and were left out in the analysis. The analysis only involved 14 seroconversions and these were followed for a mean time of 0.40 of a year and total time at risk of 286 person years. This shows incident rate of Human Immunodeficiency Virus infection of 4.9 per 100 person years of follow up, 95 \% CI: 2.9 - 8.27. Kaplan Meier curves revealed a higher risk of incident infection among women who were Bacterial Vaginosis positive than the women who were Bacterial Vaginosis negative. A log rank test showed that the probability of incident infection differed among the women depending on Bacterial Vaginosis status, 
$\mathrm{X}^{2}$ value 3.8, $\mathrm{p}$ value 0.05 . Adjusting for the other variables, incident Human Immunodeficiency Virus infection was high among Bacterial Vaginosis positive women, adjusted hazard ratio $3.21 ; 95 \% \mathrm{CI} ; 0.85-12.12$, p value 0.08 , though significance was not attained. Conclusion: The study showed an association between Bacterial Vaginosis and Human Immunodeficiency Virus seroconversion risk though statistical significance was not achieved. Education on vaginal cleansing, screening and treating women with Bacterial Vaginosis could maintain normal vaginal flora and reduce their vulnerability to Human Immunodeficiency Virus.

\section{Keywords}

Bacterial Vaginosis, Human Immunodeficiency Virus Infection, Women, Soweto, South Africa

\section{Introduction}

Human Immunodeficiency Virus (HIV) is a global health problem. The prevalence of HIV continues to grow globally and women of reproductive age are the ones who are greatly affected. About 38 million people are infected worldwide and Sub-Saharan Africa has 29 million of the total affected with the problem [1]. It is estimated that $8 \%$ of all adults in Sub-Saharan Africa are HIV infected and almost $60 \%$ of these HIV infections occur among women [2]. South Africa is one of the countries with a higher HIV infection rate in Sub-Saharan Africa and the prevalence of HIV infection has been increasing significantly with young people, mainly women, having a higher risk [3]. It is estimated that 1 in 9 people are infected and a total of 5.3 million people live with the HIV virus in South Africa. In 2003, HIV prevalence reached 27.9\% among antenatal care women attendees and an estimated $11.4 \%$ of the total population is infected [4].

Bacterial Vaginosis (BV) is a clinical syndrome which brings a change in the normal vaginal flora where the normal vaginal lactobacilli flora is replaced by other organisms anaerobic in nature leading to a higher vaginal $\mathrm{pH}$ [5] and a reduction in the number of hydrogen peroxide producing lactobacilli. Its aetiology is complex with the common organisms being Gardnerella vaginalis, Mycoplasma hominis and Mobiluncus species. It is a common condition among women and the leading cause of abnormal vaginal discharge [6].

It occurs in $20 \%-50 \%$ of women in the general population worldwide and prevalence rates of $20 \%-50 \%$ have been reported in Sub-Saharan Africa [7]. Prevalence is more in women attending sexually transmitted infection (STI) clinics (40\% - 50\%), suggesting that BV has a link with sexual activity [8].

The diagnosis of $\mathrm{BV}$ can be clinical or laboratory based examination with a gram stain by Nugent scoring criteria. The clinical diagnosis is based on an increased vaginal discharge and a higher vaginal $\mathrm{pH}>4.5$ is suggestive of $\mathrm{BV}$ [9].

The Nugent score diagnosis consists of a scoring scale system that ranges from $0-10$. A scoring of $0-3$ is negative; 4 to 6 is intermediate and 7 or above is taken as positive 
for BV [9]. Gram stain with Nugent scoring is the gold standard for diagnosing BV [10].

Literature also shows that $\mathrm{BV}$ has an association with the acquisition of HIV infection in women [6] and has been suggested as a mediator of the association between intra vaginal practices and HIV infection. Vaginal cleansing and the utilization of intra vaginal herbs are also associated with BV infection [11] [12]. Other risk factors for BV include behaviours such as greater number of sexual partners, frequent vaginal intercourse, new sexual partner and less frequent use of condoms [13] [14]. These risk factors suggest that there is a link between BV and sexual activity and fuel the debate on whether BV is an STI or not.

Besides the tremendous implications BV has on public health, there has been little attention to investigate the problem [15]. Treatment of vaginal discharge by syndromic management covers BV, however there are many organisms associated with BV and it is sometimes asymptomatic, therefore identifying proper treatment is difficult and the cure rates for BV are low; 70\% - 80\% and the rates of infection recurrence are high [7].

Several cross sectional studies have shown that BV is associated with an increased risk of HIV prevalent infection; however few prospective studies have been conducted [16]-[20].

A nested case control study was conducted in South Africa among 5110 women enrolled in a cervical cancer trial to investigate the association of BV infection and susceptibility to HIV infection. BV prevalence was 46\%, there were $86 \mathrm{HIV}$ seroconversions identified in the trial and this represented an overall incidence rate of 2.1 per 100 person years. It was found in this study that diagnosis of BV on the basis of Nugent score criteria was significantly associated with increased risk of HIV seroconversion; AOR ; 2.01; 95\% CI; 1.12 - 3.62 [21].

In Malawi a prospective study was conducted to determine the association of BV and other disturbances of the vaginal flora with HIV seroconversion among pregnant and postnatal women [22]. The diagnosis of BV was done based on clinical criteria only.

The study reported that BV was significantly associated with antenatal HIV seroconversion, AOR 3.7, p, 0.04 and postnatal HIV seroconversion, ARR 2.3, p, 0.03.

Other prospective studies were conducted in Mombasa, Kenya; Malawi and a multicentre study with sites in Uganda and Zimbabwe to examine the relationship between $\mathrm{BV}$ and HIV infection acquisition. The exposure variable BV was determined by gram stain and Nugent scoring. In all these prospective studies BV was found to be a risk factor for incident HIV-1 infection [22]-[25].

Positive findings were also reported in a meta-analysis of published studies. In the studies reviewed the diagnosis of BV was based on clinical criteria only, Nugents score criteria only as well as clinical combined with Nugents score criteria. BV was found to be associated with an increased risk of HIV acquisition in HIV incidence studies, RR $1.6,95 \% \mathrm{CI} ; 1.2$ - 2.1 [26].

Diseases of the reproductive tract have been reported to be associated with the sexual transmission of HIV infection [16]. The focus of this association has been on sexually 
transmitted infections which are known biological risk factors for acquisition and transmission of HIV infection sexually. Although BV is not a classical STI as it is unlikely to be transmitted between partners, it is associated with sexual activity and the risk factors for STIs such as increased number of sexual partners. BV is associated with changes in the vaginal flora, which weaken the natural defence mechanism present in the vagina, which may predispose women to acquisition of other STIs. Despite this, few prospective studies have been done on BV as a potential female reproductive tract infection associated with the risk of HIV infection acquisition [8]. The reported magnitude of association between BV and HIV infection is variable and depends on the definition of the exposure. Exploring the determinants for HIV infection risk among women is helpful in coming up with prevention strategies. It is therefore important to critically investigate further the association of BV and acquisition of HIV infection. This analysis added further on knowledge surrounding the temporal association of BV and HIV infection acquisition.

\section{Methods}

\subsection{Description of Primary Data}

A cohort study was conducted in Soweto between Oct. 2002 and January 2005. This cohort study aimed to estimate HIV incidence, condom usage and retention in the cohort in preparation for an HIV prevention trial. Women aged 18 to 35 years were invited to participate and those who volunteered were screened for HIV and pregnancy. Additional inclusion criteria included willingness to stay in Soweto for next year and willingness to give consent. The exclusion criteria included women outside the age range of 18 - 35, HIV positive, pregnancy positive results and intending to move out of Soweto within next one year. There were 1089 potential participants screened and 750 were enrolled. Participants were followed up for a maximum period of one year if they completed the study, however participants included in this analysis were followed for a mean period of 0.40 of a year. At entry, the participants were further screened for biological factors that included sexually transmitted diseases and BV.

\subsection{Study Procedures}

\subsubsection{Clinical Procedures}

Women were seen at the study clinic every three months. At this time HIV pre and post test counseling was done as were HIV rapid tests in parallel by trained nurses in the clinic. Structured interviews were done to collect data on potential confounders. The interviews gathered information on demographic factors that included age, level of education and social economic markers. Behavioural factor data included contraceptive use, condom use at last sexual act, number of sexual partners in the past three months, type of sexual partners and vaginal practices such as cleansing and product insertion. Pelvic examinations were done at baseline, six and 12 months visits where swabs were collected from the cervix for Neisseria Gonorrhoea (NG), Chlamydia Trachomatis (CT), from the vaginal wall for BV and trichomonas vaginalis (TV). Blood for Herpes 
Simplex Virus (HSV 2) and syphilis was also collected. Both syndromically diagnosed and laboratory diagnosed sexually transmitted infections were treated.

\subsubsection{Laboratory Procedures}

BV was determined by laboratory based Nugent Score criteria, a gram stain scoring system of vaginal smears. It consists of a scoring system and ranges from 0 - 10. In this analysis a score of 7 or above was considered positive for BV. HIV infection was diagnosed by commercially available validated rapid HIV tests done in parallel; all positive and discordant tests were confirmed by a third generation Enzyme linked immunosorbent assay (ELISA). A 10\% sample of all tests was quality controlled by ELISA. NG and CT were detected using Roche Amplicor ${ }^{\circledast}$ polymerase chain reaction (PCR) and TV sample were cultured in Diamond's media and assessed qualitatively in the laboratory. Syphilis testing was done by rapid plasma reagin (RPR) and reported by titre and testing for HSV 2 was done by Focus Herpe Select ${ }^{\circledR}$ HSV 2 ELISA.

\subsection{Study Design}

This is a secondary data analysis study of a prospective cohort which was set out to investigate HIV incidence and condom use.

\subsection{Study Population}

The study population comprised HIV negative sexually active women aged 18 - 35 years in Soweto.

\subsection{Study Sample}

The study sample was a fixed one and consisted of all the sexually active HIV negative women who were enrolled in the primary cohort study. However, 683 participants were included in this analysis and had accumulated 286 woman years of follow up; this was due to the missing of BV results. These participants were followed for a mean period of 0.40 of a year. There were 14 incident HIV infections that had BV results available and were included in this analysis.

\subsection{Measurement and Data Sources for Variables}

\subsubsection{Outcome Variable}

The outcome variable of interest was HIV infection. This was measured at three monthly intervals by commercially available rapid test. Testing was done in parallel by trained nurses in the study clinic. The tests used included Determine ${ }^{\oplus}$, Unigold ${ }^{\mathrm{Tm}}$ and Serocon.

Tests were changed due to logistic problems with supply. Each time a new test was introduced a confirmation of test performance was done by a third generation ELISA. Throughout the study all dual positive and any discordant results were confirmed by a third generation ELISA in the laboratory. In addition quality control ELISA tests were done on the first 100 rapid tests and on a $10 \%$ continuous quality control confirmation 
of rapid results.

\subsubsection{Primary Exposure Variable}

The primary exposure variable, BV, was also determined at baseline and at the six and twelve month follow up visits, gram stain was taken from the vaginal wall by a swab, read in the laboratory qualitatively and scored by Nugent's scoring. For this analysis the exposure was considered as a categorical variable of the presence of BV by Nugent score of 7 , greater than 7 or not.

\subsection{Data Processing and Data Analysis Plan}

Data cleaning and all the data analysis was done using STATA ${ }^{\bullet}$ version 10 . The required variables were extracted from the primary data set and then appropriately coded. Standard data cleaning procedures to identify values on variables was carried out by conducting checks on all the extracted variables to determine the extent of missing values and all the variable values entered as 9, 98 and 99 for example were set as missing. Participants with missing BV results were excluded from the analysis. Descriptive and analytical analysis was done to investigate the association of BV with HIV incident infection.

\subsubsection{Descriptive Statistics}

The analysis was done using STATA ${ }^{\oplus}$ version 10 and all the variable values and measures of effect have been reported with $95 \%$ confidence intervals and p values where appropriate. The descriptive frequency of social demographic and behavioural characteristics of study participants were calculated using contingency tables. The baseline prevalence of BV and STIs in the sample was also calculated using contingency tables. The chi square test of significance was conducted on categorical data and a Student's T test on continuous variables to compare the baseline characteristics of women who were positive for BV and those who were negative.

\subsubsection{Analytical Statistics}

To investigate the association of BV and HIV infection acquisition the following analysis was done.

HIV incidence was calculated; the incidence was estimated as the number of seroconversions and is expressed per 100 person years of follow up. Duration of follow up time at risk was defined as the midpoint time from enrolment to time of seroconversion for those who seroconverted and from time of enrolment to last time of follow up or last HIV negative result for those who remained negative. The risk factors for seroconversion analyzed in the models include age, income, education status, marital status, number of sexual partners last 3 months, condom use at last sexual act, vaginal cleansing, contraception use, BV, Nugent score, NG, CT, HSV 2, TV and syphilis. Kaplan Meier survival time analysis was performed comparing time to event of exposed to unexposed and log rank test for significance in the time to event were calculated. A univariate Cox regression analysis to determine whether exposure to $\mathrm{BV}$ is associated with 
HIV infection was done:

$$
H R(t)=\frac{h o(t) \exp (\beta 1)}{h o(t)}=\exp (\beta 1)
$$

The variables were then included in a multivariate Cox regression analysis with a set $p$ value of $\leq 0.1$ as a point of entry to include potential confounders. Product terms of vaginal cleansing and BV, STI and BV were run respectively to check for possible effect modification of these factors on the association between BV and HIV infection.

\subsection{Ethical Considerations}

The study protocol for secondary data analysis was assessed by the University of the Witwatersrand Human Research Ethics committee for ethical review and clearance. It was approved and its ethics clearance number is M080984. Data analysis was done only after the approval of the protocol by the ethics committee.

\section{Results}

\subsection{Social Demographic and Behavioural Characteristics of the Participants and BV Baseline Prevalence}

There were 1089 potential participants screened and 750 were enrolled in a primary cohort study following HIV negative results. The participants were all from Soweto Township and had comparable baseline characteristics. The study participants had a median age of 23 with the inter quartile range of 20 - 28 years. The majority of the participants, $48 \%(n=362)$ had 12 years or more of schooling, followed by those who had less than 12 years of schooling, $43 \%(n=320)$ and then those who did not go to school, $9 \%(n=68)$. They were mainly living in formal type of houses, $88 \%(n=662)$ and more than half, $66 \%(n=491)$ of the participants had a source of income. A large proportion of the participants, $79 \%(n=594)$ reported ever having used a condom, and only, $7 \%$ ( $n$ $=51$ ) of the participants reported to have been practicing vaginal cleansing. The majority of the participants, $82 \%(n=612)$ were not living with a sexual partner and only, $8 \%$ $(n=53)$ of the women reported having more than one sexual partner. The participants made use of contraception with, 57\% $(n=425)$ using hormonal contraception (injectable and oral contraception) and $43 \%(n=325)$, were on non hormonal contraception (condoms and natural methods) (Table 1).

The women who tested positive for BV at baseline were not statistically different from the women who tested negative for BV within the specific participants' characteristics. The overall baseline prevalence of $\mathrm{BV}$ in the study was 52\%, 95\% CI; 45 - 59. There does not seem to be any differences between those participants who had their BV results and those who had BV results missing, suggesting that BV results were missing at random. Most common reason for missing BV results was that samples were not taken at the time of the clinical visit because the participant was menstruating.

\subsection{Baseline STI Prevalence of the Study Participants}

Herpes Simplex Virus 2 had the highest prevalence in the study sample, $54 \%(n=393)$. 
This was followed by CT, TV, syphilis and NG with the prevalence of $12 \%(\mathrm{n}=83), 4 \%$ $(\mathrm{n}=31), 2 \%(\mathrm{n}=18)$ and $2 \%(\mathrm{n}=15)$ respectively (Table 2$)$.

Table 1.Description of study participants and baseline BV prevalence.

\begin{tabular}{|c|c|c|c|c|}
\hline Characteristic & n (\%) & $\mathrm{BV}$ at Baseline $\mathrm{n}(\%)$ & No BV at Baseline $\mathrm{n}(\%)$ & P Value \\
\hline \multicolumn{5}{|l|}{ Education $(n=750)$} \\
\hline No Schooling & $68(9)$ & $32(8)$ & $32(9)$ & 0.42 \\
\hline$<12$ yrs schooling & $320(43)$ & $171(45)$ & $139(40)$ & \\
\hline$>-12$ yrs school & $362(48)$ & $177(47)$ & $175(51)$ & \\
\hline \multicolumn{5}{|l|}{ House $(n=750)$} \\
\hline Formal & $662(88)$ & $334(88)$ & $310(90)$ & 0.47 \\
\hline Informal & $88(12)$ & $46(12)$ & $36(10)$ & \\
\hline \multicolumn{5}{|l|}{ Income $(n=748)$} \\
\hline Yes & $491(66)$ & $248(65)$ & $230(67)$ & 0.65 \\
\hline No & $257(34)$ & $132(35)$ & $114(33)$ & \\
\hline \multicolumn{5}{|c|}{$\begin{array}{l}\text { Condom Use last sex act } \\
\qquad(\mathrm{n}=750)\end{array}$} \\
\hline Yes & $594(79)$ & $307(81)$ & $268(77)$ & 0.26 \\
\hline No & $158(21)$ & $73(19)$ & $78(23)$ & \\
\hline \multicolumn{5}{|l|}{$\begin{array}{l}\text { Ever vaginal cleansed } \\
\qquad(\mathrm{n}=750)\end{array}$} \\
\hline Yes & $51(07)$ & $29(08)$ & $22(06)$ & 0.5 \\
\hline No & $699(93)$ & $351(92)$ & $324(94)$ & \\
\hline \multicolumn{5}{|l|}{$\begin{array}{l}\text { Number of Sexual } \\
\text { Partners last 3mths } \\
\quad(n=691)\end{array}$} \\
\hline One & $638(92)$ & $323(91)$ & $297(94)$ & 0.19 \\
\hline More than one & $53(08)$ & $32(09)$ & $20(06)$ & \\
\hline \multicolumn{5}{|c|}{ Marital Status $(n=750)$} \\
\hline Cohabiting & $138(18)$ & $71(19)$ & $61(18)$ & 0.71 \\
\hline Not Cohabiting & $612(82)$ & $309(81)$ & $285(82)$ & \\
\hline \multicolumn{5}{|c|}{ Contraception $(n=750)$} \\
\hline Hormonal & $425(57)$ & $218(57)$ & $194(56)$ & 0.72 \\
\hline Non Hormonal & $325(43)$ & $162(43)$ & $152(44)$ & \\
\hline
\end{tabular}

Table 2. Baseline STI prevalence.

\begin{tabular}{cc}
\hline Sexually Transmitted Infections & $\mathbf{n}(\%)$ \\
\hline N. gonorrhoea $(\mathrm{n}=685)$ & $15(2)$ \\
C. trachomatis $(\mathrm{n}=688)$ & $83(12)$ \\
T. vaginalis $(\mathrm{n}=737)$ & $31(4)$ \\
Herpes Simplex Virus $2(\mathrm{n}=733)$ & $393(54)$ \\
Syphilis $(\mathrm{n}=747)$ & $18(2)$ \\
\hline
\end{tabular}




\subsection{HIV Incidence and Survival Time of the Women}

There were 683 participants included in the final analysis on HIV incidence and the association between BV and HIV infection acquisition. Participants were followed for a possible maximum period of one year and were followed for a mean period of time of 0.40 of a year. This accumulated 286 person years of follow up time at risk for the participants who had BV results. There were $21 \mathrm{HIV}$ seroconversions experienced throughout the follow up period of which 7 had BV results missing and were excluded from this analysis. The remaining 14 incident infections represents the incident HIV infection rate of 4.9 per 100 person years of follow up, 95\% CI: 2.9 - 8.27. There were 4 HIV seroconversions among the women who were BV negative within a total follow up period of 161 person years. This represented an HIV incidence rate of 2.49 per 100 person years of follow up, 95\% CI: 0.93 - 6.63. Likewise, among the women who were BV positive 10 HIV seroconversions were experienced within 125 person years of follow up. The HIV incidence rate among this group was 8.0 per 100 person years of follow up, 95 \% CI: 4.3 - 14.87.

\subsubsection{Survival Time Analysis}

The Kaplan Meier survival curves for HIV seroconversion comparing women who had BV to those who did not was performed (Figure 1).

A higher risk of HIV-1 acquisition was present among women who tested positive for BV than the women who tested negative for BV at every point throughout the follow up period Figure 1. A Log rank test was performed with a chi-square value of 3.8 and $\mathrm{p}$ value 0.05 , meaning that the probability of seroconversion was different among the women depending on BV status.

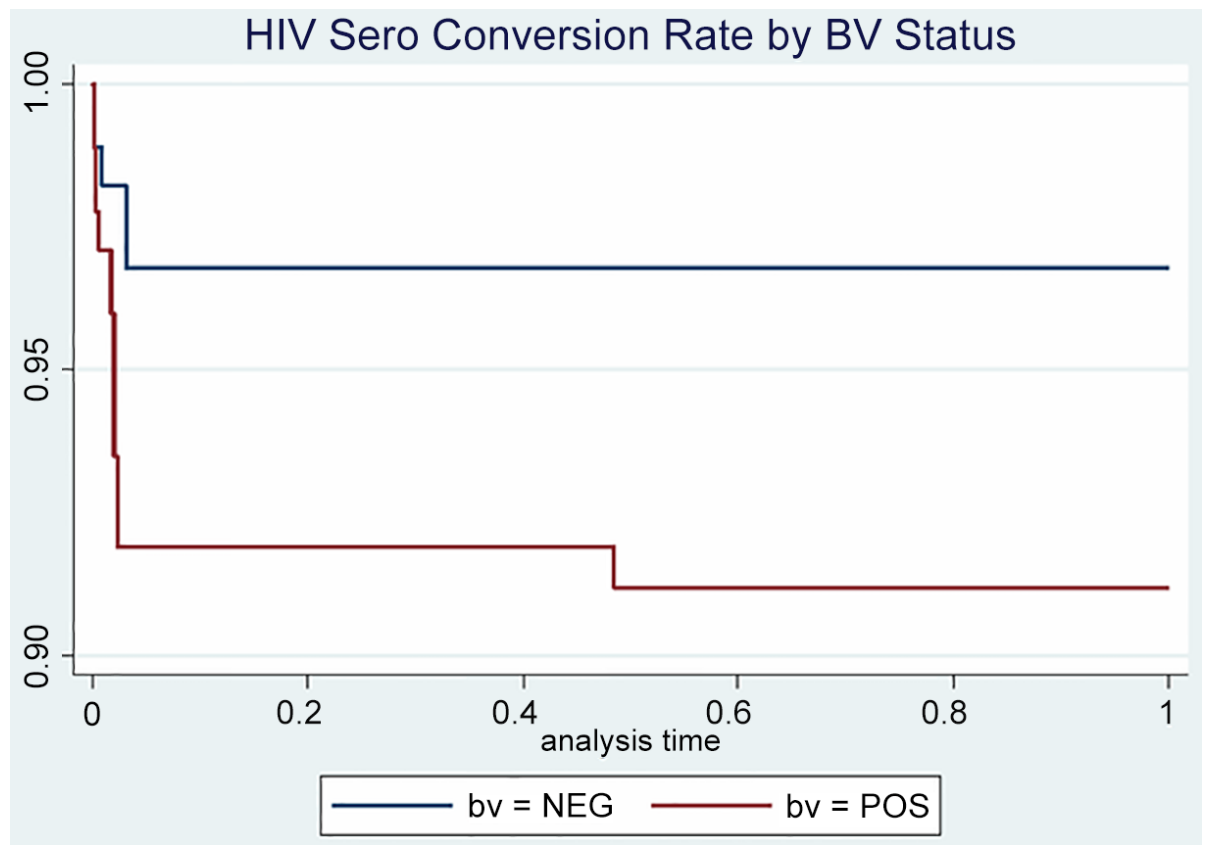

Figure 1. HIV Sero Conversion Rate by BV Status. 


\subsection{Association of BV with HIV Seroconversion}

The following factors were investigated for possible association with the risk of HIV seroconversion, BV, education level, age, income, contraception, marital status, number of sexual partners last three months, vaginal cleansing, BV Nugent score, NG, CT, and HSV 2, however, most of these did not meet the criteria for retention into the multivariate model. Vaginal cleansing is also associated with BV and may concurrently increase the risk of HIV-1 seroconversion. To assess this synergistic effect, an interaction term of BV and vaginal cleansing was run and it revealed no effect modification on the risk of HIV-1 seroconversion between BV and vaginal cleansing. An interaction term was also run for STIs and BV and there was no effect modification observed.

\subsubsection{Univariate Results}

BV infection was associated with higher risk of HIV-1 infection acquisition though statistical significance was not achieved, women who were BV positive had an increased risk of acquiring HIV-1 infection, HR, 2.97; 95\% CI, 0.93 - 9.52, p, 0.07 where as the risk of HIV-1 seroconversion for BV Nugent score was 1.2; 95\% CI: 0.98 - 1.49, p; 0.08.

The results are suggestive of a decreasing trend in the risk of HIV seroconversion as the education level of the participants increased though statistical significance was not achieved, with the women who had 12 years or more of education being less likely to seroconvert than women with less than 12 years of education, HR, 1.58; 95\% CI; 0.2 12.52 , p, 0.66, and 2.28; 95\% CI; $0.29-17.7$, p, 0.42 respectively. Similarly participants age had no statistical significance on the risk of HIV seroconversion despite its slight positive trend towards an association, HR, 1.02; 95\% CI; $0.94-1.11, \mathrm{p}, 0.56$. Likewise women who had a source of income were $52 \%$ less likely to seroconvert than the women who had no source of income, though non-significant, HR, 0.48; 95\% CI; 0.20 - 1.12, p, 0.09 .

The findings also show that vaginal cleansing could be associated with an increased risk of HIV-1 acquisition, hazard ratio, 1.95; 95\% CI; 0.26 - 14.6, p, 0.51. The women who practiced vaginal cleansing were more likely to seroconvert than those who did not. The participant's number of sexual partners could be associated with increased risk of HIV-1 seroconversion, HR; 1.94; 95\% CI; $0.44-8.44, \mathrm{P}, 0.37$ and that not living with a sexual partner may be associated with an increased risk of HIV-1 seroconversion, HR, 1.33; 95\% CI, 0.39 - 4.52; p, 0.64 .

Though statistical significance was not attained, women who were on hormonal contraception were more likely to seroconvert than women on non hormonal contraception, HR, 1.57; 95\% CI; 0.63 - 3.88, p; 0.33.

The women who had NG infection had a statistically significantly higher risk of HIV-1 seroconversion, HR, 7.4; 95\% CI, 1.61 - 33.67, p, 0.01. Similarly, CT infection was also associated with a statistically significantly increased risk of acquiring HIV infection, HR, 3.45; 95\% CI; 1.04 - 11.5, p, 0.04. The women who had HSV2 were 2.22 times, 95\% CI, $0.70-7.09$, p, 0.17, more likely to seroconvert than the women who had no HSV 2; however statistical significance was not achieved in this case. 


\subsubsection{Multivariate Results}

The variables gained entry into the multivariate model if they had a $\mathrm{p}$ of $\leq 0.1$ in the univariate model, these included BV, BV Nugent score, income, NG and CT. Variables were retained in the multivariate model if they maintained a $\mathrm{p}$ of $\leq 0.1$. The final model contained the variables BV and gonorrhoea. Therefore, after adjusting for other factors, BV was associated with an increased risk of HIV seroconversion, HR, 3.21; 95\% CI; $0.85-12.12, \mathrm{p}, 0.08$.

\section{Discussion}

This study produced no significant findings; however the findings suggest that BV is associated with an increased risk of HIV infection acquisition in women. The study revealed a high baseline BV prevalence among women aged 18 - 35 years in Soweto. The BV baseline prevalence was 52\%; 95\% CI; $45-59$ a finding which is similar to that of other studies in South Africa and many settings [5] [7] [8] [21]. The women who were $\mathrm{BV}$ positive were not statistically different from the women who were BV negative in any baseline demographic characteristics. This may be due to the fact that the women volunteered to participate in the primary study as such this made them to be similar in many aspects. The homogeneity could also be a result of the study population which only comprised women from a local South African setting in Soweto. The baseline STI prevalences in this study are similar to those reported in other studies [21] [22]. The results also shows a high HIV incidence rate of 4.9 per 100 person years of follow up, 95\% CI; 2.9 - 8.27. This is relatively higher if compared to HIV incidence estimates reported in other areas in South Africa [27]. The majority of women in this study were not living with their sexual partners and had an increased risk of HIV seroconversion, HR, 1.33, 95\% CI; $0.39-4.52$, p, 0.64. In a study to measure HIV incidence in a rural area of South Africa in KwaZulu Natal, an HIV incidence rate of 3.8 per 100 person years of follow up, 95\% CI; 3.2 - 4.6 [27] was reported.

Similarly, the HIV risk of seroconversion in the KwaZulu Natal study was twice as high among women who were unmarried but had a sexual partner than those who were married. Not living with a sexual partner, may have increased the risk of HIV seroconversion among women in this analysis as they may have been more likely to engage in risky sexual behaviours and have frequent sexual partner changes as may their partners, these factors may have increased the risk of BV infection among the women as well. High rates of infection in this case may therefore be attributed to high rates of partner change among the women. A similar HIV incidence rate of 4.51 per 100 person years of follow up, 95\% CI; 2.96 - 6.06 was reported in Malawi among women of reproductive age [24]. It was reported in the study that BV was a strong predictor of HIV infection acquisition. Likewise the results of this analysis, show the same pattern that women who were BV positive were more likely to seroconvert than the women who were BV negative, HR, 2.97, 95\% CI; 0.93 - 9.52, p, 0.07. BV may affect HIV-1 acquisition in many ways; these include vaginal $\mathrm{pH}$ changes, changes on the integrity of the vaginal epithelium and changes in the genital microflora that occur with BV [25]. These changes may increase the women's risk of HIV infection acquisition. 
After adjusting for demographic factors, other STIs and sexual behaviours, it was observed that BV was associated with the risk of HIV seroconversion, adjusted hazard ratio $3.21,95 \% \mathrm{CI} ; 0.85-12.12$, $\mathrm{p}$ value 0.08 . This suggest that $\mathrm{BV}$ is associated with a higher risk of HIV-1 infection acquisition with the BV positive women being 3 times more likely to seroconvert than the women who were BV negative within the follow up period. However, the confidence interval is wide $(0.85-12.12)$, meaning that the population value may be anywhere in between 0.85 and 12.12. In addition the confidence interval includes one and the $\mathrm{p}$ value is greater than 0.05 , implying that the association is not statistically significant. The reasons for this wide confidence interval include sample size which was small and few incident infections that were included in the analysis due to the exclusion of 7 incident infections.

These results of BV being associated with an increased risk of HIV infection acquisition are also reflected in the findings of other studies conducted in different settings on the temporal effect of the association of BV and HIV infection. A prospective study conducted in Malawi reported a significant association of BV with the risk of HIV seroconversion, an adjusted risk ratio for BV of 2.3, p; 0.03 was reported [22]. A similar pattern was also found in another prospective study done in Mombasa, Kenya [23], BV was found to be significantly associated with HIV incident infections with an adjusted hazard ratio of 1.9, 95\% CI; 1.1 - 3.2. Likewise findings obtained recently in a prospective study of HIV-1 incidence among women of reproductive age in Malawi, an association between BV and HIV infection acquisition was observed, adjusted hazard ratio, 2.52, 95\% CI; 1.07 - 5.94; p; 0.03 [24].

Positive findings were also obtained in a multi centre prospective study conducted in Zimbabwe and Uganda, BV was found statistically and significantly associated with HIV infection acquisition, AHR 1.67, 95\% CI; 1.24 - 2.26 [25]. Similarly, other positive findings were reported in a meta-analysis to assess the extent to which BV may increase the risk of HIV acquisition. BV was statistically and significantly associated with HIV infection acquisition, ARR 1.6, 95\% CI; 1.2 - 2.1 [26].

Statistical significance was not achieved in this analysis possibly due to a small number of the BV positive women who seroconverted within a total person time of follow up of 125 person years whereas the women who were BV negative who seroconverted were followed up for a total person time of 161 person years. Lack of statistical significance could also be explained as a result of the potential bias introduced by the exclusion of the 7 HIV seroconversions in this analysis which had no BV results. If this study is compared to the Malawi study [24] which had a similar sample size, but had far more HIV seroconversions analysed than those analysed in this analysis thereby achieving statistical significance. The effect size of the association of BV with the risk of HIV seroconversion is also relatively higher in this analysis than that reported in many studies [22]-[25].

This could result from a wide range of factors; firstly the prevalence of BV in the study participants though comparable to other settings was relatively higher. In a high BV prevalence setting it may be difficult to detect differences in seroconversion rates. 
Besides this, most of the participants too were not living with a sexual partner; this could be associated with frequent change of new sexual partners and hence increased risk of BV and STIs among the women and hence increased risk of HIV infection. These factors may have inflated the effect size of BV with the risk of HIV seroconversion.

The risk described in this study supports the biological plausibility of the factors described in the relationship of BV and HIV-1 acquisition. Many factors may lead to HIV-1 infection acquisition in women with BV. The normal vaginal flora which plays a protective role in women's susceptibility to sexually transmitted infections is disturbed; there is depletion of lactobacilli which may limit production of hydrogen peroxide which are protective against the organisms such as those that cause sexually transmitted infections and possibly HIV. The low vaginal $\mathrm{pH}$ has been suggested to inhibit CD4 lymphocyte activation and reduce HIV target cells in the vagina [22]. In this case lack of lactic acid produced by lactobacilli could lead to an elevated $\mathrm{pH}$ that is conducive to the growth and survival of the virus. BV also increases HIV-1 acquisition by changing cervicovaginal epithelial integrity and permeability and it is also suggested that it causes an inflammatory reaction in the female genital tract [25]. With these mechanisms present in BV positive women their risk of HIV infection may be increased.

\section{Strengths of the Study}

The primary study was a cohort study which allowed the exposure (BV) to have been measured prior to the outcome (HIV) and its temporal sequence adds to the growing knowledge of BV being associated with the risk of HIV infection acquisition among women. The primary exposure in the study was defined by laboratory Nugent score criteria. This criterion is classified as the gold standard for the diagnosis of BV [10]. This reduced measurement error and misclassification of the exposure.

The definition of the outcome too was confirmed by ELISA which has a high specificity [28]. This made it possible to precisely classify participants who truly had the HIV virus as outcomes, this as well reduced misclassification of the outcome. The primary cohort study too had a good follow up and retention rate, above $80 \%$, and in addition, the data used in this analysis is local South African data from the general population.

\section{Limitations of the Study}

The interpretation of the findings in this study is subject to important limitations.

\subsection{Volunteers}

The participants in the primary cohort study volunteered to take part as such they may differ with those who did not take part. This may have an influence on the generalizability of these findings due to selection bias this may have introduced.

\subsection{Information Bias}

Although interviewers were trained to conduct structured interviews for the initial co- 
hort, interviews can introduce information bias, and there is a possibility that participants may have hidden some pertinent information for this study.

\subsection{Secondary Data Analysis}

This study is a secondary data analysis of a primary cohort study that was designed for the evaluation of another research question and not the association between BV and the risk of HIV infection acquisition, therefore BV was not specifically taken as an exposure of interest and some information in this case was not available which was pertinent for the evaluation of this research question. For example, BV results for seven participants who seroconverted were missing and these were not included in the analysis. This may have biased the study findings.

\subsection{Few Number of End Points Analysed}

The number of HIV seroconversions analysed was low due to the exclusion of those that had no BV results; this made it difficult to find the statistical significance of the association of BV and HIV infection acquisition and made the confidence intervals wider.

\subsection{Misclassification of End Points}

The confirmation of HIV status at baseline did not include PCR testing to determine the virus prior to an antibody response; this may have misclassified already HIV positive women at baseline as HIV negative, thereby inflating seroconversion rate in the study.

\subsection{Loss to Follow up}

Though the retention rate was high, above $80 \%$ in the primary study, the women who were lost to follow up may differ considerably to the other participants in the study; this may affect generalization of the study findings. In addition some outcomes may have been missed due to lost to follow up.

\section{Recommendations}

Based on the limitations of the study, the following recommendations are proposed.

Another study designed specifically to answer the question of whether BV is associated with HIV-1 acquisition be conducted, with an adequate sample size and powered to detect a difference in the number of seroconversions between the two groups. The study should take BV as the primary exposure of interest and gather all the information regarding BV results on all the participants. A study describing the association between the treatment of BV and HIV-1 infection acquisition would also be of interest.

\section{Conclusion}

In summary, BV is an ongoing burden among women, evident with its high prevalence in this cohort. This study contributes to the knowledge that BV is associated with HIV infection acquisition. The women who were BV positive were more likely to serocon- 
vert than the women who were BV negative, although not significant. The vaginal discharge caused by BV may interrupt the lives of many women, so improved care should be provided to screen and treat BV in the primary health care settings. Women should also be exposed to education on vaginal cleansing as this predisposes them to BV.

\section{Acknowledgements}

Thanks should go to the Faculty Members, School of Public Health, Witwatersrand University for the guidance when writing the report.

\section{Conflict of Interest}

Author has no conflict of interest in respect with this research, publication and authorship of this article.

\section{References}

[1] Joint United Nations Programme on HIV/AIDS (2004) Report on the Global AIDS Epidemic. UNAIDS, Geneva.

[2] Department of Health (2004) Report on the National HIV and Syphilis Antenatal Seroprevalence Survey in South Africa, 2003. Department of Health Directorate, Health Systems Research, Research Coordination and Epidemiology, Pretoria.

[3] Pettifor, A.E., Rees, H.V., Kleinschmidt, I., Steffenson, A., Macphail, C., Hlongwa-Madikizela, L., et al. (2005) Young People's Sexual Health in South Africa: HIV Prevalence and Sexual Behaviours from a Nationally Representative House-Hold Survey. AIDS, 19, 1525 1534. http://dx.doi.org/10.1097/01.aids.0000183129.16830.06

[4] Connolly, C., Shisana, O., Colvin, M. and Stoker, D. (2004) Epidemiology of HIV in South Africa-Results of a National, Community Based Survey. South African Medical Journal, 94, 776-781.

[5] Govender, L., Hoosen, A.A., Moodley, J., Moodley, P. and Sturm, A.W. (1996) Bacterial Vaginosis and Associated Infections in Pregnancy. International Journal of Gynecology and Obstetrics, 55, 23-28. http://dx.doi.org/10.1016/0020-7292(96)02744-0

[6] Josey, W.E. and Schwebke, J.R. (2008) The Polymicrobial Hypothesis of Bacterial Vaginosis Causation: A Reassessment. International Journal of STD and AIDS, 19, 152-154. http://dx.doi.org/10.1258/ijsa.2007.007260

[7] Mayaud, P. (1997) Tackling Bacterial Vaginosis and HIV in Developing Countries. The Lancet, 350, 530-531. http://dx.doi.org/10.1016/S0140-6736(97)22034-9

[8] Schwebke, J.R. (2005) Abnormal Vaginal Flora as a Biological Risk factor for Acquisition of HIV Infection and Sexually Transmitted Disease. Journal of Infectious Diseases, 192, 13151317. http://dx.doi.org/10.1086/462430

[9] Culhane, J.F., Desanto, D., Goldenberg, R.L., McCollum, K.F., King, F. and Guaschino, S. (2005) Variation in Nugent Score and Leukocyte Count in Fluid Collected From Different Vaginal Sites. Obstetrics and Gynecology, 105, 120-123.

[10] Gutman, R.E., Peipert, J.F., Weitzen, S. and Blume, J. (2005) Evaluation of Clinical Methods for Diagnosing Bacterial Vaginosis. Obstetrics and Gynecology, 105, 551-556.

[11] Schwebke, J.R. and Desmond, R.A. (2004) Predictors of Bacterial Vaginosis in Adolescent Women Who Douche. Sexually Transmitted Diseases, 31, 433-436.

http://dx.doi.org/10.1097/01.OLQ.0000129948.91055.9F 
[12] Mbizvo, M.E., Musya, S.E., Pedersen, S.B., Chirenje, Z. and Hussein, A. (2004) Bacterial Vaginosis and Intravaginal Practices: Association with HIV. Central African Journal of Medicine, 50, 41-46.

[13] Schwebke, J.R., Richey, C.M. and Weiss, H.L. (1999) Correlation of Behaviors with Microbiological Changes in Vaginal Flora. Journal of Infectious Diseases, 180, 1632-1636. http://dx.doi.org/10.1086/315065

[14] Bradshaw, C.S., Morton, A.N., Garland, S.M., Morris, M.B., Moss, L.M. and Fairley, C.K. (2005) Higher-Risk Behavioral Practices Associated with Bacterial Vaginosis Compared with Vaginal Candidiasis. Obstetrics \& Gynecology, 106, 105-114. http://dx.doi.org/10.1097/01.AOG.0000163247.78533.7b

[15] Kaufman, R.H., Faro, S. and Brown, D. (2005) Benign Diseases of the Vulva and Vagina. Houston, Texas, 372-383.

[16] Royce, R., Sena, A. and Cates, W.J. (1997) Sexual Transmission of HIV. The New England Journal of Medicine, 336, 72-78. http://dx.doi.org/10.1056/NEJM199704103361507

[17] Cohen, R.C., Duerr, A., Pruithithada, N., Rugpao, S., Hillier, S., Garcia, P., et al. (1995) Bacterial Vaginosis and HIV Seroprevalence among Female Sex Workers in Chiang Mai Thailand. AIDS, 9, 1093-1097. http://dx.doi.org/10.1097/00002030-199509000-00017

[18] Sewankambo, N., Gray, R.H., Wawer, M.J., Paxton, L., McNairn, D., Wabwire-Mengen, F., et al. (1997) HIV-1 Infection Associated with Abnormal Vaginal Flora Morphology and Bacterial Vaginosis. The Lancet, 350, 546-550.

http://dx.doi.org/10.1016/S0140-6736(97)01063-5

[19] Royce, R.A., Thorp, J., Granados, J.L. and Savtz, D.A. (1999) Bacterial Vaginosis Associated with HIV in Pregnant Women from North Carolina. Journal of Acquired Immune Deficiency Syndromes, 20, 383-386. http://dx.doi.org/10.1097/00042560-199904010-00009

[20] Moodley, P., Connolly, C. and Sturm, W. (2002) Interrelationships among Human Immunodeficiency Virus Type 1 Infection, Bacterial Vaginosis, Trichomoniasis, and the Presence of Yeasts. Journal of Infectious Diseases, 185, 69-73. http://dx.doi.org/10.1086/338027

[21] Myer, L., Denny, L., Telerant, R., Souza, M.D., Wright, T.C. and Kuhn, L. (2005) Bacterial Vaginosis and Susceptibility to HIV Infection in South African Women: A Nested CaseControl Study. Journal of Infectious Diseases, 192, 1372-1380.

http://dx.doi.org/10.1086/462427

[22] Taha, T.E., Hoover, D.R., Dallabetta, G.A., Kumwenda, N.I., Mtimavalye, L.A.R., Yang, L., et al. (1998) Bacterial Vaginosis and Disturbances of Vaginal Flora: Association with increased acquisition of HIV. AIDS, 12, 1699-1706. http://dx.doi.org/10.1097/00002030-199813000-00019

[23] Martin, H.L., Richardson, B.A., Nyange, P.M., Lavreys, L., Hillier, S.L., Chohan, B., et al. (1999) Vaginal Lactobacilli, Microbial Flora, and Risk of Human Immunodeficiency Virus Type 1 and Sexually Transmitted Disease Acquisition. Journal of Infectious Diseases, 180, 1863-1868. http://dx.doi.org/10.1086/315127

[24] Kumwenda, N.I., Kumwenda, J., Kafulafula, G., Makanani, B., Taulo, F., Nkhoma, C., et al. (2008) HIV-1 Incidence among Women of Reproductive Age in Malawi. International Journal of STD \& AIDS, 19, 339-341. http://dx.doi.org/10.1258/ijsa.2007.007165

[25] Van de Wijgert, J.H.H.M., Morrison, C.S., Cornelisse, P.G.A., Munjoma, M., Moncada, J., Awio, P., et al. (2008) Bacterial Vaginosis and Vaginal Yeast, but Not Vaginal Cleansing, Increase HIV-1 Acquisition in African Women. Journal of Acquired Immune Deficiency Syndromes, 48, 203-210. http://dx.doi.org/10.1097/QAI.0b013e3181743936

[26] Atashili, J., Poole, C., Ndumbe, P.M., Adimora, A.A. and Smith, J.S. (2008) Bacterial Vagi- 
nosis and HIV Acquisition: A Meta-Analysis of Published Studies. AIDS, 22, 1493-1501. http://dx.doi.org/10.1097/QAD.0b013e3283021a37

[27] Barnighausen, T.A.B., Tanser, F.A., Gqwede, Z.A., Mbizana, C.A., Herbst, K.A. and Newell, M.A.C. (2008) High HIV Incidence in a Community with High HIV Prevalence in Rural South Africa: Findings from a Prospective Population Based Study. AIDS, 22, 139-144. http://dx.doi.org/10.1097/QAD.0b013e3282f2ef43

[28] Constantine, N.T., Saville, R.D. and Dax, E.M. (2005) Retroviral Testing and Quality Assurance: Essentials for Laboratory Diagnosis. Malloy Printers, Ann Arbor.

\section{Submit or recommend next manuscript to SCIRP and we will provide best service} for you:

Accepting pre-submission inquiries through Email, Facebook, LinkedIn, Twitter, etc. A wide selection of journals (inclusive of 9 subjects, more than 200 journals)

Providing 24-hour high-quality service

User-friendly online submission system

Fair and swift peer-review system

Efficient typesetting and proofreading procedure

Display of the result of downloads and visits, as well as the number of cited articles

Maximum dissemination of your research work

Submit your manuscript at: http://papersubmission.scirp.org/

Or contact ojpm@scirp.org 